\title{
Astrophysically important reactions involving excited hydrogen
}

Cite as: AIP Conference Proceedings 312, 437 (1994); https:// doi.org/10.1063/1.46568

Published Online: 12 May 2008

J. M. C. Rawlings, J. E. Drew, and M. J. Barlow

\section{ARTICLES YOU MAY BE INTERESTED IN}

Spontaneous emission lifetimes in the ground electronic states of $\mathrm{HD}^{+}$and $\mathrm{H}_{2}{ }^{+}$

The Journal of Chemical Physics 71, 5382 (1979); https:// doi.org/10.1063/1.438353

\section{Lock-in Amplifiers up to $600 \mathrm{MHz}$}
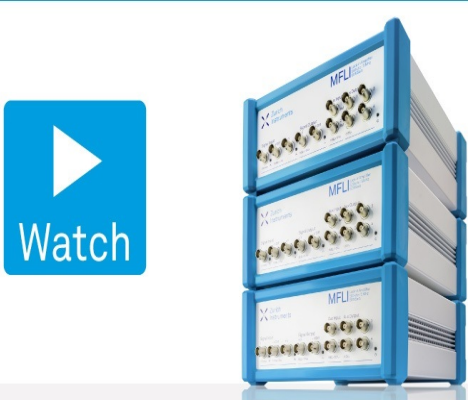


\title{
ASTROPHYSICALLY IMPORTANT REACTIONS INVOLVING EXCITED HYDROGEN
}

\author{
J.M.C. Rawlings \\ Department of Mathematics, UMIST, Manchester, UK
}

J.E. Drew

Department of Physics, Astrophysics, University of Oxford, Oxford, UK

M.J. Barlow

Department of Physics and Astronomy, University College London, London, UK

\begin{abstract}
The associative ionization reaction $\mathrm{H}(\mathrm{n}=2)+\mathrm{H} \rightarrow \mathrm{H}_{2}^{+}+\mathrm{e}^{-}$is found to be a greater contributor to the $\mathrm{H}_{2}$ formation rate than the direct radiative association reaction $\mathrm{H}(\mathbf{n}=2)+\mathrm{H} \rightarrow \mathrm{H}_{2}+\mathrm{h} \nu$ in most regions of astrophysical interest. The endothermicity $(\simeq 1.1 \mathrm{eV})$ of the reaction and the high departures from LTE that are required for the H I $(\mathbf{n}=2)$ level to be sufficiently populated restrict its significance to regions of high excitation. The reaction $\mathrm{H}(\mathrm{n}=2)+\mathrm{H}^{+} \rightarrow \mathrm{H}_{2}^{+}+\mathrm{h} \nu$ may be significant in highly excited ionized regions, such as planetary nebulae and shocks. Chemical models of circumstellar regions have been reassessed in the light of this information. A critical examination reveals that excitation effects are, in general, very important in many astrophysical situations. Only exceptionally, will reactions involving the higher excited states $(n>2)$ be as significant as those involving $\mathrm{H}(\mathrm{n}=2)$.
\end{abstract}

\section{INTRODUCTION}

The chemistry of circumstellar and nebular regions, where temperatures and densities are high and the radiation field strong, differs greatly from that which is applicable to the general interstellar medium. In particular, the formation of $\mathrm{H}_{2}$ would be expected to be severely inhibited. Yet there are examples of such environments where it has been detected (e.g. in certain planetary nebulae).

The well-established pathways to $\mathrm{H}_{2}$ formation in the gas phase are as follows. First, there is the direct three-body reaction:

$$
\mathrm{H}+\mathrm{H}+\mathrm{H} \longrightarrow \mathrm{H}_{2}+\mathrm{H}
$$

For this reaction to be significant the density must typically be greater than about $10^{11}$ $\mathrm{cm}^{-3}$. At lower densities and moderate ionization levels a much more efficient route is via the $\mathrm{H}^{-}$ion:

$$
\begin{aligned}
& \mathrm{H}+e^{-} \longrightarrow \mathrm{H}^{-}+h \nu \\
& \mathrm{H}^{-}+\mathrm{H} \longrightarrow \mathrm{H}_{2}+e^{-}
\end{aligned}
$$

However, the $\mathrm{H}^{-}$ion can be lost by mutual neutralisation and is susceptible to photodetachment by the radiation field even at near-infrared wavelengths. 


\section{Reactions Involving Excited Hydrogen}

Finally, as a 'last resort' there is the much less efficient $\mathrm{H}^{+}$route:

$$
\begin{aligned}
& \mathrm{H}^{+}+\mathrm{H} \longrightarrow \mathrm{H}_{2}^{+}+h \nu \\
& \mathrm{H}_{2}^{+}+\mathrm{H} \longrightarrow \mathrm{H}_{2}+\mathrm{H}^{+}
\end{aligned}
$$

Again, the intermediate, $\mathrm{H}_{2}^{+}$is susceptible to photodissociation and dissociative recombination.

Previously, it has not been common practise to take into proper account the internal excitation of the reacting chemical species. If the relevant downward transitions are highly optically thick then trapping may occur (as is often the case for H I Ly $\alpha$ ) and the higher states may be well populated. A critical examination reveals that excitation effects are, in general, very important in many astrophysical situations.

\section{RADIATIVE ASSOCIATION}

Latter and Black ${ }^{1}$ proposed that $\mathrm{H}_{2}$ formation may occur as a result of direct radiative association;

$$
\mathrm{H}+\mathrm{H}(n=2) \longrightarrow \mathrm{H}_{2}+h \nu
$$

The rate coefficient for this reaction was calculated to be $1.2 / 1.1 \times 10^{-14} \mathrm{~cm}^{3} \mathrm{~s}^{-1}$ at $1000 / 10000 \mathrm{~K}$ for the $2 \mathrm{~s}$ channel and $3.4 / 4.2 \times 10^{-14} \mathrm{~cm}^{3} \mathrm{~s}^{-1}$ at $1000 / 10000 \mathrm{~K}$ for the $2 p$ channel. Latter and Black investigated the recombination era in the early Universe $(z>1000)$, diffuse HII regions and cool protostellar winds (as modelled by Glassgold et. al. ${ }^{2,3}$ ). Only in the first of these did the new process make a significant difference to the production of $\mathrm{H}_{2}$.

\section{ASSOCIATIVE IONIZATION}

Associative ionization is the most elementary collisional process by which molecular bonds can be formed. The cross-section for the associative ionization reaction:-

$$
\mathrm{H}+\mathrm{H}(2 s) \longrightarrow \mathrm{H}_{2}^{+}+e^{-}
$$

has been measured by Urbain et al. ${ }^{4}$. The measured threshold for this reaction is about $1.1 \mathrm{eV}$. The $2 \mathrm{p}$ channel cannot, as yet, be measured directly owing to the short radiative lifetime of the state. We have assumed that the sum of the $2 \mathrm{~s}$ and the $2 \mathrm{p}$ processes results in a smooth, featureless cross-section.

The calculated rate coefficient for the reaction (from Rawlings, Drew and Barlow ${ }^{5}$ ) are given in Table 1 for the $2 \mathrm{~s}$ channel using the measured cross-sections, for the $2 \mathrm{p}$ channel using our inferred cross-sections and for the $\mathrm{n}=2$ weighted mean cross-section (assuming statisitical weight populations).

The temperature dependences of the rate coefficients are smooth and fits to them are given by:

$$
\begin{aligned}
k_{2 s}=1.16 \times 10^{-10} T^{-0.07} e^{-16941 / T} \mathrm{~cm}^{3} \mathrm{~s}^{-1} & (3800 \leq T \leq 21800 \mathrm{~K}) \\
k_{2 p}=1.19 \times 10^{-8} T^{-0.5} e^{-19065 / T} \mathrm{~cm}^{3} \mathrm{~s}^{-1} & (3400 \leq T \leq 22000 \mathrm{~K}) \\
k_{2 s, p}=2.41 \times 10^{-9} T^{-0.35} e^{-17829 / T} \mathrm{~cm}^{3} \mathrm{~s}^{-1} & (3000 \leq T \leq 22000 \mathrm{~K})
\end{aligned}
$$

These are accurate to within $10 \%$ over the specified temperature ranges. 
Table 1: Values of the rate coefficient $\left(\mathrm{cm}^{3} \mathrm{~s}^{-1}\right)$ for the associative ionization reaction for several temperature values

\begin{tabular}{cccc}
\hline Temperature $(\mathrm{K})$ & $\mathrm{H}(2 \mathrm{~s})$ & $\mathrm{H}(2 \mathrm{p})$ & $\mathrm{H}(2 \mathrm{~s}, \mathrm{p})$ \\
\hline & & & \\
3000 & $3.1 \times 10^{-13}$ & $4.6 \times 10^{-13}$ & $4.2 \times 10^{-13}$ \\
5000 & $2.1 \times 10^{-12}$ & $3.7 \times 10^{-12}$ & $3.3 \times 10^{-12}$ \\
10000 & $1.11 \times 10^{-11}$ & $1.78 \times 10^{-11}$ & $1.61 \times 10^{-11}$ \\
15000 & $1.69 \times 10^{-11}$ & $2.73 \times 10^{-11}$ & $2.51 \times 10^{-11}$ \\
\hline
\end{tabular}

The reaction is followed by charge exchange (re. 5) to form $\mathrm{H}_{2}$. Although this route again involves the unstable intermediate $\mathrm{H}_{2}^{+}$, the measured cross-sections are substantially larger than those for the radiative association reaction. Hence, except at lower temperatures $(<6000 \mathrm{~K})$ this reaction is of wider significance.

Associative ionization is unlikely to compete with the negative ion route in circumstances in which $\mathrm{H}^{-}$easily survives. However, there are situations in which associative ionization is favoured over the positive ion radiative association route. Over the probable temperature range of interest, $5000 \lesssim T \lesssim 10000 \mathrm{~K}, n_{2} / n_{+}$must be in excess of $1-3$ $\times 10^{-5}$ for associative ionization to dominate.

At the densities typical of the situations where $\mathrm{H}_{2}$ formation is an issue, large departures from LTE are therefore required before associative ionization dominates. Such environments are typically less than half-ionized, cooler than $10000 \mathrm{~K}$ and with a high Lyman- $\alpha$ optical depth.

\section{EXCITED HYDROGEN CHEMISTRY}

If a true assessment is to be made of the importance of these reactions and related pathways, we must consider the effects that the excitation of molecular as well as atomic species has on the chemistry.

To describe a pure hydrogen gas experiencing a significant degree of excitation in a non-equilibrium environment, the effect of radiation transport in at least a two-level atom must be treated. The generation and transfer of H I Ly $\alpha$ and two-photon emission must be included, both in the treatment of the hydrogen atom and in the photochemistry of the more complex molecular species. Listed below are some of the reactions whose high energy behaviour has been analysed in some detail as part of these investigations.

- $\mathrm{H}+\mathrm{H}^{+} \longrightarrow \mathrm{H}_{2}^{+}+\mathrm{h} \nu$

- $\mathrm{H}^{-}+\mathrm{e}^{-} \longrightarrow \mathrm{H}+\mathrm{e}^{-}+\mathrm{e}^{-}$

- $\mathrm{H}_{2}\left(\mathrm{v}^{\prime \prime} \geq 0\right)+\mathrm{h} \nu \longrightarrow \mathrm{H}+\mathrm{H}$

- $\mathrm{H}_{2}{ }^{\star}+\mathrm{h} \nu \longrightarrow \mathrm{H}_{2}^{+}+\mathrm{e}^{-}$

- $\mathrm{H}_{2}^{+\star}+\mathrm{h} \nu \longrightarrow \mathrm{H}^{+}+\mathrm{H}$

- $\mathrm{H}_{2}^{+\star}+\mathrm{e}^{-} \longrightarrow \mathrm{H}+\mathrm{H}$

- Collisional dissociations of $\mathrm{CO}, \mathrm{H}_{2}, \mathrm{~N}_{2}, \mathrm{C}_{2}, \mathrm{O}_{2}$ and other molecules which have a small or zero dipole moment 
FURTHER REACTIONS INVOLVING EXCITED ATOMIC HYDROGEN

The corresponding associative ionization reaction for $H(n=3)$

$$
\mathrm{H}+\mathrm{H}(n=3) \rightarrow \mathrm{H}_{2}^{+}+e^{-}
$$

is exothermic by $\simeq 1.14 \mathrm{eV}$. However, the $\mathrm{n}=3$ level is $\simeq 1.89 \mathrm{eV}$ above $\mathrm{n}=2$ and will have a correspondingly lower population in nearly all regions of interest. Calculations of the cross-section by X.Urbain (private communication) imply a rate-coefficient of;

$$
k \simeq\left(2.1 \times 10^{-7} / T\right) e^{-7137 / T} \mathrm{~cm}^{-3} \mathrm{~s}^{-1} \quad \text { for } 4400 \leq \mathrm{T} \leq 22000 \mathrm{~K}
$$

Over the temperature range $4500-8000 \mathrm{~K}$ this is never more than about four times as large as the corresponding rate for the $n=2$ reaction and to exceed the corresponding $\mathrm{n}=2$ rate, marked population inversion is required. The reaction:

$$
\mathrm{H}(n=3)+\mathrm{H} \longrightarrow \mathrm{H}_{2}+h \nu
$$

may also need to be considered in certain circumstances, but as yet no data are available.

Urbain, Cornet \& Jureta ${ }^{6}$ have measured the cross-section for the reaction:

$$
\mathrm{H}(2 s)+\mathrm{H}(2 s) \longrightarrow \mathrm{H}_{2}^{+}+e^{-}
$$

while Poulaert et al. ${ }^{7}$ have measured, and Urbain et al. ${ }^{8}$ have calculated, the cross-section for the reaction:

$$
\mathrm{H}^{+}+\mathrm{H}^{-} \longrightarrow \mathrm{H}_{2}^{+}+e^{-}
$$

The values are found to be some 10 to 100 times as large as the calculated values for the $\mathrm{H}(\mathrm{n}=2)$ associative ionization reaction at the collision energies of interest. However, it is not expected that these reactions will be important since they will be in direct competition with other reactions discussed above.

Of greater potential interest is the excited state analogue of the radiative association reaction (4):

$$
\mathrm{H}^{+}+\mathrm{H}(n=2) \longrightarrow \mathrm{H}_{2}^{+}+h \nu
$$

Preliminary estimates indicate that $\mathrm{k} \sim 4 \times 10^{-13} \mathrm{~cm}^{3} \mathrm{~s}^{-1}$ in the temperature range of interest, in which case the reaction may be significant in regions of high ionization (such as recombination zones, old PN etc.).

\section{APPLICATIONS TO ASTROPHYSICAL MODELS}

Excited hydrogen chemistry has so far been considered only in immediate circumstellar environments, focussing on the winds of young stellar objects ${ }^{5}$ :

1. winds from massive young stellar objects

2. T Tauri winds

3. cool neutral outflows

We are currently working on a variety of other environments ${ }^{9}$ including; 
1. old planetary nebulae

2. supernovae (and remnants)

3. shocks

4. the recombination epoch of the early Universe

The basic details of the T Tauri and cool neutral outflow models can be found in Rawlings, Williams \& Canto ${ }^{10}$ and Glassgold, Mamon \& Huggins ${ }^{3}$. Particular attention is paid to the radiative transfer and the relevant continuum opacities, and their effects on the photorates. Because of the high $\mathrm{H}(\mathrm{n}=2)$ population in the regions of interest, the time-dependence of the Balmer continuum opacity is especially important.

1. Winds from massive young stellar objects.

The winds associated with massive YSOs are likely to be warm out to large radii, and are certainly dense and fairly well-ionized. The low outflow velocities $(\sim 100 \mathrm{~km}$ $\mathrm{s}^{-1}$ ) and high mass loss rates can yield significant Balmer as well as extreme Lyman continuum optical depth in the outflow. In these circumstances both the $\mathrm{H}(\mathrm{n}=2)$ population and the $\mathrm{H}: \mathrm{H}^{+}$ratio will be enhanced. In addition, many molecular species (e.g. $\mathrm{H}_{2}$ and, to an extent, $\mathrm{H}_{2}^{+}$) are protected against photodissociation.

The model assumes spherical symmetry and uses a velocity law following Castor \& Lamers $^{11}$. We use the wind temperature profile based on the OB star wind models of Drew ${ }^{12}$ (fit presented in Bunn \& Drew ${ }^{13}$ ). The emergent radiative energy distribution of the YSO was represented by the calculated spectrum from a Kurucz ${ }^{14}$ model atmosphere.

The Balmer continuum opacity is extremely sensitive to the details of the velocity law as well as to mass loss rate. Results indicate that for high mass loss rates ( $10^{-5} \mathrm{M}_{\odot} \mathrm{yr}^{-1}$ ) and slow wind accelerations the associative ionization is the dominant $\mathrm{H}_{2}$ formation channel out to about $80 \mathrm{R}_{\star}$ despite the rapid photodissociation rate for $\mathrm{H}_{2}^{+}$. In these circumstances a high $\mathrm{H}(\mathrm{n}=2)$ population can be maintained over a large radius range. Reaction (6) accounts for less than $18 \%$ of the $\mathrm{H}_{2}$ formation rate throughout the wind. The $\mathrm{H}_{2}$ abundance peaks at $1.4 \times 10^{-7}$ at a radius of about $4.3 \mathrm{R}_{\star}$. This is a factor of about six times larger than would be the case if the associative ionization reaction (7) were omitted from the chemistry.

2. T Tauri winds.

T-Tauri winds are both hot $\left(10^{4} \mathrm{~K}\right)$ and excited (with an excitation/ionization temperature peaking at $\geq 15000 \mathrm{~K}$ ). The model of Rawlings et al. ${ }^{10}$ has been adapted to accommodate the new chemistry. The physical basis of this model is described in Hartmann, Edwards \& Avrett ${ }^{15}$; Terminal velocity and maximum temperatures are reached at about $3-5 R_{\star}$. The mass loss rate is small $\left(\simeq 5 \times 10^{-9}\right.$ $\mathrm{M}_{\odot} \mathrm{yr}^{-1}$ ) and the cooling rate is large. The Balmer continuum optical depth is therefore low at all times. 


\section{Reactions Involving Excited Hydrogen}

Associative ionization is found to have a marginal significance and only within $0.1 R_{\star}$ of the starting radius $\left(5 R_{\star}\right)$. The reason for this is straightforward - because of the high excitation temperature and the (relatively) low densities, $\mathrm{H}_{2}^{+}$is more rapidly destroyed by the IR radiation field than by charge exchange to form $\mathrm{H}_{2}$. Direct excited state radiative association (6) is more important and results in a significant enhancement of $\mathrm{H}_{2}$ (by a factor of about 7 within a few $\mathrm{R}_{\star}$ ).

3. Cool neutral outflows.

In the dense, cool, neutral winds associated with low mass YSOs, such as SVS $13 / \mathrm{HH}$ $7-11$, there is no significant Lyman continuum radiation. Thus, in the inner wind, where Ly $\alpha$ is extremely optically-thick, detailed balance $\left(b_{1}=b_{2}\right)$ holds.

In order to test the importance of the associative ionization reaction and the other reactions discussed above we have investigated a model for the outflow from SVS 13 that is much like that (Case 2) considered by Glassgold, Mamon \& Huggins ${ }^{3}$.

At the lower temperatures $(5000 \mathrm{~K}) \mathrm{H}(\mathrm{n}=2)$ reactions tend to be unimportant, since detailed balance holds; but at $7500 \mathrm{~K}$ the $\mathrm{H}(\mathrm{n}=2)$ fractional abundance is over three orders of magnitude higher at radii where Ly $\alpha$ remains in detailed balance $\left(b_{1}=b_{2} \simeq 90\right)$ and a large Balmer continuum optical depth accumulates. In this setting the associative ionization $\mathrm{H}_{2}$ formation route is dominant out to about $35 \mathrm{R}_{\star}$, where the $\mathrm{H}^{+}$route takes over (re. 4,5). The $\mathrm{H}_{2}$ abundance is higher by a factor of about 3 (within $20 \mathrm{R}_{\star}$ ) as compared to the cooler model. This higher $\mathrm{H}_{2}$ abundances may be sustained in the outer parts of the wind if a realistic cooling law is employed - essentially "freezing out" the chemistry.

The outflow from SVS 13 is apparently too cool for excited state routes to $\mathrm{H}_{2}$ to be significant. However, the strong temperature dependence of the associative ionization implies that it is important in slightly warmer environments.

\section{REFERENCES}

1. Latter, W.B. and Black, J.H., Astrophys. J. 372, 161 (1991)

2. Glassgold, A.E., Mamon, G.A. and Huggins, P.J., Astrophys. J. 336, L29 (1989)

3. Glassgold, A.E., Mamon, G.A. and Huggins, P.J., Astrophys. J. 373, 254 (1991)

4. Urbain, X., Cornet, A., Brouillard, F. and Giusti-Suzor, A., Phys. Rev. Lett. 66, 1685 (1991)

5. Rawlings, J.M.C., Drew, J.E. and Barlow, M.J., Mon. Not. of the Roy. Ast. Soc. (in press) (1993)

6. Urbain, X., Cornet, A. and Jureta, J., J. Phys. B. 25, L189 (1992)

7. Poulaert, G., Brouillard, F., Claeys, W., McGowan, J.W. and Van Wassenhove, G., J. Phys. B. 11, L671 (1978)

8. Urbain, X., Giusti-Suzor, A., Fussen, D. and Kubach, C., J. Phys. B. 19, L273 (1986)

9. Barlow, M.J., Rawlings, J.M.C. and Drew, J.E., Mon. Not. of the Roy. Ast. Soc. (to be submitted)

10. Rawlings, J.M.C., Williams, D.A. and Canto, J., Mon. Not. of the Roy. Ast. Soc. 230, 695 (1988) 
11. Castor, J.I. and Lamers, H.G.J.L.M., Astrophys. J. Supp. 39, 481 (1979)

12. Drew, J.E., Astrophys. J. Supp. 71, 267 (1989)

13. Bunn, J.C. and Drew, J.E., Mon. Not. of the Roy. Ast. Soc. 255, 449 (1992)

14. Kurucz, R.L., Stellar Atmospheres: Beyond Classical Models

(NATO ASI series C Vol. 341, Kluwer Academic Publishers, 1991) p.441

15. Hartmann, L., Edwards, S. and Avrett, E., Astron. J. 261, 279 (1982) 